\title{
INTRINSIC GEOMETRY AND BOUNDARY STRUCTURE OF PLANE DOMAINS
}

\author{
OONA RAINIO, TOSHIYUKI SUGAWA, AND MATTI VUORINEN
}

\begin{abstract}
For a non-empty compact set $E$ in a proper subdomain $\Omega$ of the complex plane, we denote the diameter of $E$ and the distance from $E$ to the boundary of $\Omega$ by $d(E)$ and $d(E, \partial \Omega)$, respectively. The quantity $d(E) / d(E, \partial \Omega)$ is invariant under similarities and plays an important role in Geometric Function Theory. In the present paper, when $\Omega$ has the hyperbolic distance $h_{\Omega}(z, w)$, we consider the infimum $\kappa(\Omega)$ of the quantity $h_{\Omega}(E) / \log (1+d(E) / d(E, \partial \Omega))$ over compact subsets $E$ of $\Omega$ with at least two points, where $h_{\Omega}(E)$ stands for the hyperbolic diameter of the set $E$. We denote the upper halfplane by $\mathbb{H}$. Our main results claim that $\kappa(\Omega)$ is positive if and only if the boundary of $\Omega$ is uniformly perfect and that the inequality $\kappa(\Omega) \leq \kappa(\mathbb{H})$ holds for all $\Omega$, where equality holds precisely when $\Omega$ is convex.
\end{abstract}

\section{INTRODUCTION}

Let $\Omega$ be a domain in the complex plane $\mathbb{C}$ with the hyperbolic metric $\rho_{\Omega}(z)|d z|$ of Gaussian curvature -1 [1]. The celebrated Uniformization Theorem [2, p. 81] guarantees the existence of $\rho_{\Omega}$ for a domain $\Omega$ when its boundary $\partial \Omega$ contains at least three points. Such a domain is called hyperbolic. Here and in what follows, the boundary of a domain is taken with respect to the Riemann sphere $\widehat{\mathbb{C}}=\mathbb{C} \cup\{\infty\}$.

The function $\rho_{\Omega}(z)$ is sometimes called the hyperbolic density of $\Omega$. For instance, for the unit disk $\mathbb{D}=\{z \in \mathbb{C}:|z|<1\}$ and the upper half-plane $\mathbb{H}=\{z \in \mathbb{C}: \operatorname{Im} z>0\}$, the hyperbolic densities are given by $\rho_{\mathbb{D}}(z)=2 /\left(1-|z|^{2}\right)$ and $\rho_{\mathbb{H}}(z)=1 / \operatorname{Im} z$, respectively. Let $h_{\Omega}\left(z_{1}, z_{2}\right)$ denote the hyperbolic distance induced by $\rho_{\Omega}(z)|d z|$ and $d(z, \partial \Omega)$ the Euclidean distance from a point $z \in \Omega$ to the boundary $\partial \Omega$. Then we have the inequality $\rho_{\Omega}(z) \leq 2 / d(z, \partial \Omega)$ for each $z \in \Omega$ as a simple consequence of Schwarz' Lemma [3, (2.1)]. On the other hand, the inequality $\rho_{\Omega}(z) \geq 1 /(2 d(z, \partial \Omega))$ holds for a simply connected domain $\Omega$ [3, (2.2)], [1, p. 35 Thm 8.6], [4, p.34, (3.2.1)].

The distance on $\Omega$ induced by the continuous Riemannian metric $|d z| / d(z, \partial \Omega)$ is called the quasihyperbolic distance and denoted by $k_{\Omega}\left(z_{1}, z_{2}\right)$ [5]. We now have the inequality $h_{\Omega}\left(z_{1}, z_{2}\right) \leq 2 k_{\Omega}\left(z_{1}, z_{2}\right)$ for a general domain $\Omega$ and $h_{\Omega}\left(z_{1}, z_{2}\right) \geq k_{\Omega}\left(z_{1}, z_{2}\right) / 2$ for a simply connected domain $\Omega$. These two inequalities are very handy, because there are many

File: UP20210331.tex, printed: 2021-4-1, 1.09

2010 Mathematics Subject Classification. Primary 30F45; Secondary 30C85.

Key words and phrases. Condenser capacity, hyperbolic metric, uniformly perfect set.

The authors were supported in part by JSPS KAKENHI Grant Number JP17H02847. 
estimates for quasihyperbolic distances whereas hyperbolic distances are not easy to estimate because the density function $\rho_{\Omega}(z)$ depends on the local boundary structure in the vicinity of $z$ in a subtle manner [3], [2, p.241, Thm 14.5.2], [6]. It should be noticed that the second estimate does not apply to general domains, because the hyperbolic distance is not bounded from below by a constant multiple of the quasihyperbolic distance, for instance, if the domain has isolated boundary points. To measure the similarity between $h_{\Omega}$ and $k_{\Omega}$, the domain functional [7]

$$
c(\Omega)=\inf _{z \in \Omega} \rho_{\Omega}(z) d(z, \partial \Omega)=\inf _{z_{1}, z_{2} \in \Omega, z_{1} \neq z_{2}} \frac{h_{\Omega}\left(z_{1}, z_{2}\right)}{k_{\Omega}\left(z_{1}, z_{2}\right)}
$$

is useful, where the second equality will be proven in the next section. By the above observations, we have $c(\Omega) \leq 2$ for a general domain $\Omega$ and $c(\Omega) \geq 1 / 2$ for a simply connected domain $\Omega$. But more is known about this domain constant.

Theorem A. Let $\Omega$ be a hyperbolic domain in $\mathbb{C}$. Then $c(\Omega) \leq 1$ with equality if and only if $\Omega$ is convex. Furthermore, $c(\Omega)>0$ if and only if $\partial \Omega$ is uniformly perfect.

The general inequality $c(\Omega) \leq 1$ is due to Harmelin and Minda [7] and the equality condition is due to Mejía and Minda [8]. The last assertion is due to Beardon and Pommerenke [3. Here, a closed set $E$ in $\widehat{\mathbb{C}}$ with card $(E) \geq 2$ is said to be uniformly perfect if there is a constant $0<\alpha<1$ such that the closed annulus $\alpha r \leq|z-a| \leq r$ meets $E$ whenever $a \in E$ and $0<r<d(E)$. Here and hereafter, card $(E)$ denotes the cardinality of the set $E$ and $d(E)$ is the Euclidean diameter of $E$. In other words, $d(E)=\sup _{z, w \in E}|z-w|$. We set $d(E)=+\infty$ when $\infty \in E$. For uniformly perfect sets, we refer to [9], [10, pp. 343-345], [11], [12], [13] and [14. Uniform perfectness has many applications in potential theory, metric spaces, Kleinian groups and complex dynamics as well as geometric function theory; see, in addition to the above references, for instance [9], [15, [16] and [17].

In their work about the quasihyperbolic metric, Gehring and Palka [5] also introduced the distance-ratio metric

$$
j_{\Omega}\left(z_{1}, z_{2}\right)=\log \left(1+\frac{\left|z_{1}-z_{2}\right|}{\min \left\{d\left(z_{1}, \partial \Omega\right), d\left(z_{2}, \partial \Omega\right)\right\}}\right)
$$

for $z_{1}, z_{2} \in \Omega$, see also [18, p.61]. They proved that $j_{\Omega}\left(z_{1}, z_{2}\right) \leq k_{\Omega}\left(z_{1}, z_{2}\right)$ holds always. It is also known that $j_{\Omega}$ satisfies the triangle inequality on $\Omega$ [18, p.59, Lemma 4.6]. The opposite inequality characterises so called uniform domains: a domain $\Omega$ is uniform if and only if there exists a constant $b>0$ such that the inequality

$$
k_{\Omega}\left(z_{1}, z_{2}\right) \leq b j_{\Omega}\left(z_{1}, z_{2}\right)
$$

holds, see Gehring and Osgood [19] and [18, p.84]. These domains are ubiquitous in geometric function theory [4].

It is a natural and interesting question to ask what can be said if we replace $k_{\Omega}$ by $h_{\Omega}$. Our answer is the following result. 
Theorem 1.2. Let $\Omega$ be a hyperbolic domain in $\mathbb{C}$. There is a constant $c>0$ such that $c j_{\Omega}\left(z_{1}, z_{2}\right) \leq h_{\Omega}\left(z_{1}, z_{2}\right)$ for all $z_{1}, z_{2} \in \Omega$ if and only if the boundary of $\Omega$ in $\widehat{\mathbb{C}}$ is uniformly perfect.

In conjunction with the Gehring-Osgood theorem [19, pp.59-60], we have the following result.

Corollary 1.3. Let $\Omega$ be a hyperbolic domain in $\mathbb{C}$. Then the hyperbolic metric $h_{\Omega}$ is comparable with the distance-ratio metric $j_{\Omega}$ if and only if $\Omega$ is uniform and has uniformly perfect boundary.

Indeed, if for some constants $0<c_{1} \leq c_{2}$,

$$
c_{1} j_{\Omega}\left(z_{1}, z_{2}\right) \leq h_{\Omega}\left(z_{1}, z_{2}\right) \leq c_{2} j_{\Omega}\left(z_{1}, z_{2}\right), \quad \text { for } z_{1}, z_{2} \in \Omega
$$

we first see that $\partial \Omega$ is uniformly perfect. Then $h_{\Omega}$ is comparable with $k_{\Omega}$ by Theorem A. We now conclude that $\Omega$ is uniform by the Gehring-Osgood theorem. The converse follows readily from Theorem 1.2 and the Gehring-Osgood theorem.

For a subset $E$ of $\Omega$ with card $(E) \geq 2$, we define the set functionals

$$
h_{\Omega}(E)=\sup _{z_{1}, z_{2} \in E} h_{\Omega}\left(z_{1}, z_{2}\right) \quad \text { and } \quad J_{\Omega}(E)=\log \left(1+\frac{d(E)}{d(E, \partial \Omega)}\right) .
$$

Here and hereafter, $d(E, F)$ denotes the Euclidean distance between the sets $E$ and $F$. For a singleton $E=\{z\}$, we write $d(\{z\}, F)=d(z, F)=d(F, z)$. We will use the following monotonicity property frequently in the sequel: $h_{\Omega}(E) \leq h_{\Omega}\left(E^{\prime}\right)$ and $J_{\Omega}(E) \leq J_{\Omega}\left(E^{\prime}\right)$ for $E \subset E^{\prime} \subset \Omega$. We note that $h_{\Omega}(E)$ is the hyperbolic diameter of $E$ in $\Omega$ and that $J_{\Omega}(E)$ is important in connection with capacity estimates of $E$ (see, for instance, [20]). We now consider the domain constant

$$
\kappa(\Omega)=\inf _{E} \frac{h_{\Omega}(E)}{J_{\Omega}(E)},
$$

where $E$ runs over all compact subsets of $\Omega$ with card $(E) \geq 2$. As the following result tells, the two domain constants $c(\Omega)$ and $\kappa(\Omega)$ are comparable.

Theorem 1.4. Let $\Omega$ be a hyperbolic domain in $\mathbb{C}$. Then the double inequality

$$
\frac{c(\Omega)}{2} \leq \kappa(\Omega) \leq c(\Omega)
$$

holds. In particular, $\kappa(\Omega)>0$ if and only if $\partial \Omega$ is uniformly perfect.

It is a little surprising that the quantity $\kappa(\Omega)$ behaves like $c(\Omega)$ in the following sense (compare with Theorem A).

Theorem 1.5. Let $\Omega$ be a hyperbolic domain in $\mathbb{C}$. Then, the inequality $\kappa(\Omega) \leq \kappa(\mathbb{H})$ holds, where equality holds if and only if $\Omega$ is convex. 
In view of the above theorem, we are curious about the value of $\kappa(\mathbb{H})$. However, it seems difficult to evaluate it in a simple form. Since $c(\mathbb{H})=1$, the first part of Theorem 1.4 implies $1 / 2 \leq \kappa(\mathbb{H}) \leq 1$. We will prove later that the inequality $\kappa(\mathbb{H})<1$ holds and give a numerical approximation of the value of $\kappa(\mathbb{H})$ in Theorem 4.18, thus answering a problem formulated in [18, p.455, item (12)].

The existence of an extremal configuration of the set $E$ for the functional $h_{\Omega}(E) / J_{\Omega}(E)$ is more subtle. We will prove the following result in the final section. We note that a convex domain in $\mathbb{C}$ carries the hyperbolic metric unless it is $\mathbb{C}$ itself.

Theorem 1.6. Let $\Omega$ be a convex proper subdomain of $\mathbb{C}$. There exists a compact subset $E$ in $\Omega$ satisfying $\kappa(\Omega)=h_{\Omega}(E) / J_{\Omega}(E)$ if and only if $\Omega$ is a half-plane.

When $\Omega$ is the upper half-plane $\mathbb{H}$, there exists a three-point set $E^{*}$ of the form $\left\{i, z_{1}, z_{2}\right\}$ constituting a hyperbolic equilateral triangle with $\kappa(\mathbb{H})=h_{\mathbb{H}}\left(E^{*}\right) / J_{\mathbb{H}}\left(E^{*}\right), 1<\operatorname{Im} z_{j}(j=$ $1,2)$ and $z_{1}=-\overline{z_{2}}$. Moreover, such an extremal three-point set is unique up to similarities keeping $\mathbb{H}$ invariant.

In view of the application given in the final section, it is important to have a lower bound of $\kappa(\Omega)$ when $\Omega$ is simply connected. We consider the number

$$
\kappa_{0}=\inf _{\Omega} \kappa(\Omega)
$$

where $\Omega$ runs over all simply connected proper subdomains of $\mathbb{C}$. By Theorem 1.4 and the well-known estimate $c(\Omega) \geq 1 / 2$, we obtain $\kappa_{0} \geq 1 / 4$. On the other hand, when $\Omega$ is the slit domain $\Omega_{0}=\mathbb{C} \backslash(-\infty, 0]$, numerically we have $\kappa\left(\Omega_{0}\right) \leq h_{\Omega_{0}}(E) / J_{\Omega_{0}}(E)=0.4251604 \ldots$ for $E=\left\{w_{0}, w_{1}, w_{2}\right\}, w_{0}=1, w_{1}=2.121820474+1.198476681 i, w_{2}=\bar{w}_{1}$. Note that $h_{\Omega_{0}}\left(w_{0}, w_{1}\right)=h_{\Omega_{0}}\left(w_{0}, w_{2}\right) \approx h_{\Omega_{0}}\left(w_{1}, w_{2}\right)$. Thus, we have the following corollary.

Corollary 1.8. $1 / 4 \leq \kappa_{0}<0.4251605$.

It is an open problem to determine the value $\kappa_{0}$.

The organization of this paper is as follows. In Section 2, preliminary results cocerning the domain constant $\kappa(\Omega)$ are given and Theorems 1.2 and 1.4 are proved. Section 3 is devoted to the proof of Theorem[1.5. We determine extremal configurations of three-point sets $E$ with respect to the set functional $h_{\mathbb{H}}(E) / J_{\mathbb{H}}(E)$ and prove Theorem 1.6 in Section 4. We also give numerical observations on the quantity $\kappa(\mathbb{H})$. We will apply our results to lower estimation of the capacity of a condenser in the final section.

\section{Preliminaries}

In this section, we prove several simple preliminary results. We begin with the proof of the second equality in (1.1). To distinguish the both sides of (1.1), for a while, we write

$$
c(\Omega)=\inf _{z \in \Omega} \rho_{\Omega}(z) d(z, \partial \Omega) \quad \text { and } \quad c^{\prime}(\Omega)=\inf _{z, w \in \Omega} \frac{h_{\Omega}(z, w)}{k_{\Omega}(z, w)} .
$$


We will prove that $c(\Omega)=c^{\prime}(\Omega)$. Since $\rho_{\Omega}(z) \geq c(\Omega) / d(z, \partial \Omega)$, we easily obtain $h_{\Omega}\left(z_{1}, z_{2}\right) \geq$ $c(\Omega) k_{\Omega}\left(z_{1}, z_{2}\right)$. Hence, $c^{\prime}(\Omega) \geq c(\Omega)$. On the other hand, by the formula

$$
\lim _{w \rightarrow z} \frac{h_{\Omega}(z, w)}{k_{\Omega}(z, w)}=\lim _{w \rightarrow z} \frac{h_{\Omega}(z, w)}{|z-w|} \cdot \frac{|z-w|}{k_{\Omega}(z, w)}=\frac{\rho_{\Omega}(z)}{1 / d(z, \partial \Omega)}=\rho_{\Omega}(z) d(z, \partial \Omega),
$$

we have $c(\Omega) \geq c^{\prime}(\Omega)$. Thus, we are done.

For the analysis of domain constants, we introduce some variants of the domain constant $\kappa(\Omega)$. First, we replace $h_{\Omega}$ with $k_{\Omega}$ and define the domain constant

$$
\hat{\kappa}(\Omega)=\inf _{E} \frac{k_{\Omega}(E)}{\log (1+d(E) / d(E, \partial \Omega))},
$$

where the infimum is taken over all compact subsets $E$ of $\Omega$ with card $(E) \geq 2$. Here, $k_{\Omega}(E)$ denotes the quasihyperbolic diameter of $E$. We also define the following auxiliary domain constants for integers $n \geq 2$ :

$$
\kappa_{n}(\Omega)=\inf _{E \subset \Omega, \operatorname{card}(E)=n} \frac{h_{\Omega}(E)}{\log (1+d(E) / d(E, \partial \Omega))}
$$

and

$$
\hat{\kappa}_{n}(\Omega)=\inf _{E \subset \Omega, \operatorname{card}(E)=n} \frac{k_{\Omega}(E)}{\log (1+d(E) / d(E, \partial \Omega))} .
$$

For $E=\left\{z_{1}, \ldots, z_{n}\right\}$, letting $z_{n} \rightarrow z_{n-1}$, we observe that

$$
\kappa_{2}(\Omega) \geq \kappa_{3}(\Omega) \geq \cdots \geq \kappa(\Omega)
$$

and

$$
\hat{\kappa}_{2}(\Omega) \geq \hat{\kappa}_{3}(\Omega) \geq \cdots \geq \hat{\kappa}(\Omega) .
$$

For these domain constants, we have the following results. In particular, we see that $\kappa_{n}(\Omega)=\kappa(\Omega)$ and $\hat{\kappa}_{n}(\Omega)=\hat{\kappa}(\Omega)$ for every $n \geq 3$.

Lemma 2.1. $\quad$ (i) $\hat{\kappa}_{2}(\Omega) \geq 1$.

(ii) $\kappa_{3}(\Omega)=\kappa(\Omega)$ and $\hat{\kappa}_{3}(\Omega)=\hat{\kappa}(\Omega)$.

(iii) $\kappa_{2}(\Omega) \leq 2 \kappa_{3}(\Omega)$ and $\hat{\kappa}_{2}(\Omega) \leq 2 \hat{\kappa}_{3}(\Omega)$.

Proof. Part (i) is clear from the Gehring-Palka inequality $k_{\Omega}\left(z_{1}, z_{2}\right) \geq j_{\Omega}\left(z_{1}, z_{2}\right)$. Let $E$ be an arbitrary compact set in $\Omega$ with $\operatorname{card}(E) \geq 2$. Take $z_{0}, z_{1}, z_{2} \in E$ so that $d(E)=\left|z_{1}-z_{2}\right|$ and $d(E, \partial \Omega)=d\left(z_{0}, \partial \Omega\right)$ and let $E_{0}=\left\{z_{0}, z_{1}, z_{2}\right\}$. (Note that one of the points $z_{1}, z_{2}$ may be the same as $z_{0}$.) Then

$$
\begin{aligned}
h_{\Omega}(E) \geq h_{\Omega}\left(E_{0}\right) & \geq \kappa_{3}(\Omega) \log \left(1+d\left(E_{0}\right) / d\left(E_{0}, \partial \Omega\right)\right) \\
& =\kappa_{3}(\Omega) \log \left(1+\left|z_{1}-z_{2}\right| / d\left(z_{0}, \partial \Omega\right)\right) \\
& =\kappa_{3}(\Omega) \log (1+d(E) / d(E, \partial \Omega)) .
\end{aligned}
$$


Taking the infimum over compact subsets $E$ of $\Omega$, we obtain the inequality $\kappa(\Omega) \geq \kappa_{3}(\Omega)$. Since $\kappa(\Omega) \leq \kappa_{3}(\Omega)$ as we noted above, we conclude $\kappa(\Omega)=\kappa_{3}(\Omega)$. In the same way, we can verify $\hat{\kappa}(\Omega)=\hat{\kappa}_{3}(\Omega)$.

Finally, we prove part (iii). Let $E \subset \Omega$ with card $(E)=3$ and choose $z_{0} \in E$ so that $d(E, \partial \Omega)=d\left(z_{0}, \partial \Omega\right)$. Also choose $z_{1}, z_{2} \in E$ so that $d(E)=\left|z_{1}-z_{2}\right|$. Then

$$
\begin{aligned}
& \log (1+d(E) / d(E, \partial \Omega)) \\
= & \log \left(1+\left|z_{1}-z_{2}\right| / d\left(z_{0}, \partial \Omega\right)\right) \\
\leq & \log \left(1+\left(\left|z_{1}-z_{0}\right|+\left|z_{2}-z_{0}\right|\right) / d\left(z_{0}, \partial \Omega\right)\right) \\
\leq & \log \left(1+\left|z_{1}-z_{0}\right| / d\left(z_{0}, \partial \Omega\right)\right)+\log \left(1+\left|z_{2}-z_{0}\right| / d\left(z_{0}, \partial \Omega\right)\right) \\
\leq & \kappa_{2}(\Omega)^{-1}\left(h_{\Omega}\left(z_{1}, z_{0}\right)+h_{\Omega}\left(z_{2}, z_{0}\right)\right) \\
\leq & 2 h_{\Omega}(E) / \kappa_{2}(\Omega),
\end{aligned}
$$

which implies $\kappa_{2}(\Omega) \leq 2 \kappa_{3}(\Omega)$. In the same way, we can prove the other inequality.

We need also the following simple lemma.

Lemma 2.3. For a hyperbolic domain $\Omega$ in $\mathbb{C}$, the inequality $\kappa_{2}(\Omega) \leq c(\Omega)$ holds.

Proof. Noting the formula

$$
\lim _{w \rightarrow z} \frac{h_{\Omega}(z, w)}{j_{\Omega}(z, w)}=\rho_{\Omega}(z) d(z, \partial \Omega)
$$

we have

$$
\kappa_{2}(\Omega)=\inf _{z \neq w} \frac{h_{\Omega}(z, w)}{j_{\Omega}(z, w)} \leq \inf _{z \neq w} \rho_{\Omega}(z) d(z, \partial \Omega)=c(\Omega) .
$$

We are now in a position to prove Theorem 1.4.

Proof of Theorem 1.4. By the above lemma and the inequality $h_{\Omega}(x, y) \geq c(\Omega) k_{\Omega}(x, y)$, for an arbitrary compact set $E$ in $\Omega$, we have

$$
\begin{aligned}
\frac{h_{\Omega}(E)}{\log (1+d(E) / d(E, \partial \Omega))} & \geq \frac{c(\Omega) k_{\Omega}(E)}{\log (1+d(E) / d(E, \partial \Omega))} \\
& \geq c(\Omega) \hat{\kappa}(\Omega) \geq \frac{c(\Omega)}{2} \hat{\kappa}_{2}(\Omega) \geq \frac{c(\Omega)}{2} .
\end{aligned}
$$

Hence we have $\kappa(\Omega) \geq c(\Omega) / 2$. The other inequality follows from Lemma 2.3 ;

$$
\kappa(\Omega) \leq \kappa_{2}(\Omega) \leq c(\Omega) .
$$

We now prove Theorem 1.2 . 
Proof of Theorem 1.2. Assume that $c j_{\Omega}\left(z_{1}, z_{2}\right) \leq h_{\Omega}\left(z_{1}, z_{2}\right)$ for $z_{1}, z_{2} \in \Omega$. Then $\kappa_{2}(\Omega) \geq c$. By Lemma 2.1 and Theorem 1.4, we obtain

$$
c(\Omega) \geq \kappa(\Omega) \geq \frac{1}{2} \kappa_{2}(\Omega) \geq \frac{c}{2}>0 .
$$

Thus, $\partial \Omega$ is uniformly perfect. Conversely, if $\partial \Omega$ is uniformly perfect, similarly we obtain $\kappa_{2}(\Omega) \geq \kappa(\Omega) \geq c(\Omega) / 2>0$. Thus, $c j_{\Omega}\left(z_{1}, z_{2}\right) \leq h_{\Omega}\left(z_{1}, z_{2}\right)$ holds with $c=\kappa_{2}(\Omega)>0$.

\section{Proof of Theorem 1.5}

In this section, we will prove Theorem 1.5 step by step. We begin with the following result.

Lemma 3.1. For any hyperbolic domain $\Omega$ in $\mathbb{C}$, the inequality $\kappa(\Omega) \leq \kappa(\mathbb{D})$ holds.

Proof. By definition, for a given $\varepsilon>0$, there is a compact subset $E$ of $\mathbb{D}$ such that

$$
\frac{h_{\mathbb{D}}(E)}{J_{\mathbb{D}}(E)}<\kappa(\mathbb{D})+\varepsilon .
$$

Moreover, by rotating $E$ if necessary, we may further assume that the nearest point of the boundary $\partial \mathbb{D}$ to $E$ is 1 . Namely, $d(E, \partial \mathbb{D})=d(E, 1)$.

Let $\Omega$ be an arbitrary hyperbolic domain in $\mathbb{C}$. For an arbitrarily fixed point $z_{0} \in \Omega$, choose $\zeta_{0} \in \partial \Omega$ so that $d\left(z_{0}, \partial \Omega\right)=\left|z_{0}-\zeta_{0}\right|$. Since $\kappa(\Omega)$ is invariant under similarities, we may assume that $z_{0}=0$ and $\zeta_{0}=1$. Then $\mathbb{D} \subset \Omega$. By the domain monotonicity of the hyperbolic metric, we have $h_{\Omega}(E) \leq h_{\mathbb{D}}(E)$. On the other hand, we have $d(E, \partial \Omega)=$ $d(E, 1)=d(E, \partial \mathbb{D})$ so that $J_{\Omega}(E)=J_{\mathbb{D}}(E)$. Hence,

$$
\kappa(\mathbb{D})+\varepsilon>\frac{h_{\mathbb{D}}(E)}{J_{\mathbb{D}}(E)} \geq \frac{h_{\Omega}(E)}{J_{\Omega}(E)} \geq \kappa(\Omega) .
$$

Since $\varepsilon>0$ is arbitrary, we obtain the required inequality $\kappa(\mathbb{D}) \geq \kappa(\Omega)$.

Remark 3.2. Note that the set functional $J_{D}(E)$ in the above proof is not the same thing as the diameter of $E$ in the $j_{D}$ metric

$$
j_{D}(E)=\sup \left\{j_{D}(x, y): x, y \in E\right\} .
$$

It is easy to see that the inequality

$$
J_{D}(E) / 2 \leq j_{D}(E) \leq J_{D}(E)
$$

holds for all $E \subset D$, with equality in the second inequality if $E$ is a disk, card $(E)=2$, or card $(E)=3$ and the triangle with vertices $E$ is either equilateral or a so-called Reuleaux triangle.

Moreover, for a half-plane, we have the following result.

Lemma 3.3. Let $H$ be an open half-plane in $\mathbb{C}$. Then $\kappa(\mathbb{D})=\kappa(H)$. 
Proof. By Lemma 3.1, it is enough to prove the inequality $\kappa(H) \geq \kappa(\mathbb{D})$. We choose the right half-plane $\{z: \operatorname{Re} z>0\}$ as $H$. For every $\varepsilon>0$, we can find a compact subset $E$ of $H$ such that

$$
\frac{h_{H}(E)}{J_{H}(E)}<\kappa(H)+\varepsilon
$$

Let $\zeta_{0}$ be the nearest boundary point to $E$. For simplicity, we assume that $\zeta_{0}=0$. For $R>0$, we denote the disk $\{z:|z-R|<R\}$ by $\Delta_{R}$. For a large enough $R, E \subset \Delta_{R}$ and $d\left(E, \partial \Delta_{R}\right)=d(E, 0)=d(E, \partial H)$ so that $J_{H}(E)=J_{\Delta_{R}}(E)$. On the other hand, since

$$
\rho_{\Delta_{R}}(z)=\frac{2 R}{R^{2}-|z-R|^{2}}=\frac{1}{\operatorname{Re} z-|z|^{2} /(2 R)} \rightarrow \frac{1}{\operatorname{Re} z}=\rho_{H}(z)
$$

locally uniformly on $\mathbb{H}$, we obtain $h_{\Delta_{R}}(E) \rightarrow h_{H}(E)$ as $R \rightarrow+\infty$. Noting the inequality

$$
h_{\Delta_{R}}(E) / J_{\Delta_{R}}(E) \geq \kappa\left(\Delta_{R}\right)=\kappa(\mathbb{D}),
$$

we have

$$
\frac{h_{H}(E)}{J_{H}(E)}=\lim _{R \rightarrow+\infty} \frac{h_{\Delta_{R}}(E)}{J_{\Delta_{R}}(E)} \geq \kappa(\mathbb{D}) .
$$

Hence, $\kappa(H)+\varepsilon>\kappa(\mathbb{D})$. Since $\varepsilon>0$ was arbitrary, we obtain the inequality $\kappa(H) \geq \kappa(\mathbb{D})$ as required.

We next prove the following lemma.

Lemma 3.4. Let $\Omega$ be a convex domain in $\mathbb{C}$ with $\Omega \neq \mathbb{C}$. Then $\kappa(\Omega)=\kappa(\mathbb{D})$.

Proof. Let $E$ be any compact subset of $\Omega$. Take $\zeta_{0} \in \partial \Omega$ so that $d(E, \partial \Omega)=d\left(E, \zeta_{0}\right)$. Since $\Omega$ is convex, there is a supporting line, say, $L$ at the point $\zeta_{0}$. Let $H$ be the connected component of $\mathbb{C} \backslash L$ containing $\Omega$. Then $\Omega \subset H$ and $\zeta_{0} \in \partial H=L$. Since $d(E, \partial H)=$ $d\left(E, \zeta_{0}\right)=d(E, \partial \Omega)$, we obtain

$$
\frac{h_{\Omega}(E)}{J_{\Omega}(E)} \geq \frac{h_{H}(E)}{J_{H}(E)} \geq \kappa(H)=\kappa(\mathbb{D}) .
$$

Here, we used Lemma 3.3. Taking the infimum over $E$, we obtain the inequality $\kappa(\Omega) \geq$ $\kappa(\mathbb{D})$. Recalling Lemma 3.1, we have the desired relation.

To deduce the equality condition is the most subtle part in the proof of Theorem 1.5. A key ingredient is Keogh's lemma about non-convex domains. See Figure 1.

Lemma 3.5 (Keogh [21]). Suppose that a domain $\Omega$ in $\mathbb{C}$ is not convex. Then there are two open disks $\Delta_{1}$ and $\Delta_{2}$ whose boundaries intersect perpendicularly such that $G=$ $\Delta_{1} \backslash \overline{\Delta_{2}}$ is contained in $\Omega$ and the midpoint $\zeta_{0}$ of the concave boundary arc $\Delta_{1} \cap \partial \Delta_{2}$ of $G$ lies on the boundary $\partial \Omega$ of $\Omega$.

We are now ready to prove the following result, which is the last piece of the proof of Theorem 1.5. 


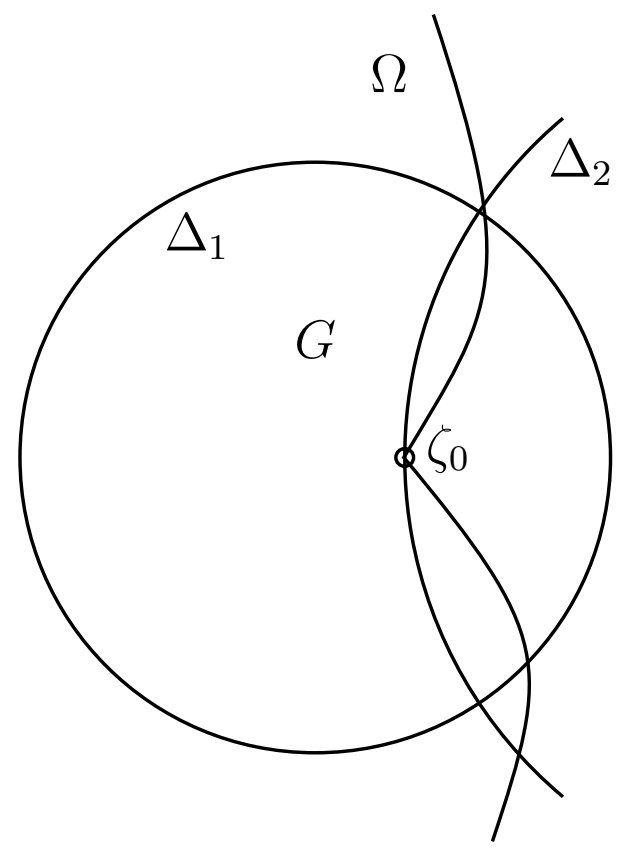

Figure 1. The domain $G=\Delta_{1} \backslash \overline{\Delta_{2}}$ in $\Omega$

Lemma 3.6. Let $\Omega$ be a non-convex domain in $\mathbb{C}$. Then $\kappa(\Omega)<\kappa(\mathbb{D})$.

Proof. We find open disks $\Delta_{1}, \Delta_{2}$ as in Keogh's lemma so that $G=\Delta_{1} \backslash \overline{\Delta_{2}} \subset \Omega$ and the midpoint $\zeta_{0}$ of the concave boundary arc of $G$ is contained in $\partial \Omega$. We may assume that $\Delta_{1}=\mathbb{D}$ and $\zeta_{0}=a \in(0,1)$ so that the center of $\Delta_{2}$ lies on the real axis. Then the second disk $\Delta_{2}$ is the image of the right half-plane $H$ under the Möbius transformation

$$
T(z)=\frac{z+a}{1+a z}
$$

Thus, $G=T\left(\mathbb{D}_{-}\right)$, where $\mathbb{D}_{-}$is the left half $\{z \in \mathbb{D}: \operatorname{Re} z<0\}$ of the unit disk. We now construct a conformal map $f$ of the upper half-plane $\mathbb{H}$ onto $G$ as follows. We denote the analytic automorphism $(1+z / 2) /(1-z / 2)$ of $\mathbb{H}$ by $M$. Note that $M$ maps the positive imaginary axis $i \mathbb{R}_{+}=\{i y: 0<y<+\infty\}$ onto the upper half of the unit circle $|\zeta|=1$. The function $S(\zeta)=\sqrt{\zeta}$ maps $\mathbb{H}$ onto the first quadrant $D=\{w: \operatorname{Re} w>0, \operatorname{Im} w>0\}$. Then the Möbius transformation $L(w)=i(w-1) /(w+1)$ maps $D$ onto the left half $\mathbb{D}_{\text {_ of }} \mathbb{D}$. Hence, the function $f=T \circ L \circ S \circ M$ maps $\mathbb{H}$ onto $G$ in such a way that $f\left(i \mathbb{R}_{+}\right)=(-1, a)$. More concretely, $f$ is expressed by

$$
f(z)=T\left(i \frac{\sqrt{1+z / 2}-\sqrt{1-z / 2}}{\sqrt{1+z / 2}+\sqrt{1-z / 2}}\right) .
$$


In view of this form, we see that $f(z)$ is analytic on $|z|<1$. (This follows also from the Schwarz reflection principle.) Therefore, we can expand $f(z)$ about $z=0$ as follows:

$$
f(z)=a+a_{1} z+a_{2} z^{2}+\cdots \quad(|z|<1) .
$$

By a straightforward computation, we have here

$$
a_{1}=\frac{i}{4}\left(1-a^{2}\right), \quad a_{2}=\frac{a}{16}\left(1-a^{2}\right)
$$

and therefore

$$
A:=\frac{a_{2}}{a_{1}}=\frac{a}{4 i} .
$$

Let $E_{x}:=x E^{*}=\left\{x z_{j}: j=0,1,2\right\}$ for $0<x<1$, where $E^{*}=\left\{z_{0}, z_{1}, z_{2}\right\} \subset \mathbb{H}$ with $z_{0}=i$ is the set in Theorem 1.6 and thus $\kappa(\mathbb{H})=h_{\mathbb{H}}\left(E^{*}\right) / J_{\mathbb{H}}\left(E^{*}\right)$. Let $w_{j}=f\left(x z_{j}\right)$ and set $E_{x}^{\prime}=f\left(E_{x}\right)=\left\{w_{j}: j=0,1,2\right\}$. Since $f(x z)=a+a_{1} x z+O\left(x^{2}\right)$ as $x \rightarrow 0$ locally uniformly in $z, d\left(E_{x}^{\prime}\right)=\left|w_{1}-w_{2}\right|$ and $d\left(E_{x}^{\prime}, \partial G\right)=d\left(w_{0}, \partial G\right)=d\left(w_{0}, \Delta_{1} \cap \partial \Delta_{2}\right)$ for a small enough $x>0$. Note here that $w_{0}=f\left(x z_{0}\right)=f(i x) \in(0, a)$ because $f\left(i \mathbb{R}_{+}\right)=(-1, a)$. Hence, $d\left(E_{x}^{\prime}, \partial G\right)=d\left(w_{0}, \Delta_{1} \cap \partial \Delta_{2}\right)=d\left(w_{0}, a\right)$. We now look at the quantity

$$
F(x)=\frac{d\left(E_{x}^{\prime}\right)}{d\left(E_{x}^{\prime}, \partial G\right)}=\frac{\left|w_{1}-w_{2}\right|}{\left|w_{0}-a\right|}=\left|\frac{w_{1}-w_{2}}{w_{0}-a}\right| .
$$

We observe that

$$
W=\frac{w_{1}-w_{2}}{w_{0}-a}=\frac{f\left(x z_{1}\right)-f\left(x z_{2}\right)}{f\left(x z_{0}\right)-f(0)}
$$

is even analytic in $x \in \mathbb{D}$ and we compute

$$
\begin{aligned}
W & =\frac{a_{1} x\left(z_{1}-z_{2}\right)+a_{2} x^{2}\left(z_{1}^{2}-z_{2}^{2}\right)+O\left(x^{3}\right)}{a_{1} x z_{0}+a_{2} x^{2} z_{0}^{2}+O\left(x^{3}\right)} \\
& =\frac{z_{1}-z_{2}}{z_{0}} \cdot \frac{1+A x\left(z_{1}+z_{2}\right)+O\left(x^{2}\right)}{1+A x z_{0}+O\left(x^{2}\right)} \\
& =\frac{z_{1}-z_{2}}{z_{0}} \cdot\left[1+A x\left(z_{1}+z_{2}-z_{0}\right)+O\left(x^{2}\right)\right],
\end{aligned}
$$

where $A=a_{2} / a_{1}=a /(4 i)$ by (3.7). Hence $F(x)=|W|$ is real analytic in $-1<x<1$ and

$$
\begin{aligned}
F(x) & =\frac{\left|z_{1}-z_{2}\right|}{\left|z_{0}\right|}\left\{1+\operatorname{Re}\left[A x\left(z_{1}+z_{2}-z_{0}\right)\right]+O\left(x^{2}\right)\right\} \\
& =\frac{\left|z_{1}-z_{2}\right|}{\left|z_{0}\right|}\left\{1+\frac{a x}{4} \operatorname{Im}\left(z_{1}+z_{2}-z_{0}\right)+O\left(x^{2}\right)\right\}
\end{aligned}
$$

as $x \rightarrow 0$. Since $\operatorname{Im} z_{j}=d\left(z_{j}, \partial \mathbb{H}\right)>d\left(z_{0}, \partial \mathbb{H}\right)$ for $j=1,2$, we have

$$
F(0)=\frac{\left|z_{1}-z_{2}\right|}{\left|z_{0}\right|}=\frac{d\left(E^{*}\right)}{d\left(E^{*}, \partial \mathbb{H}\right)} \quad \text { and } \quad F^{\prime}(0)=\frac{a\left|z_{1}-z_{2}\right|}{4\left|z_{0}\right|} \operatorname{Im}\left(z_{1}+z_{2}-z_{0}\right)>0 .
$$


In particular, $F(x)$ is strictly increasing at $x=0$ and thus $F(x)>F(0)$ for small enough $x>0$. Since $G \subset \Omega$, we have the inequality $h_{\Omega}\left(E_{x}^{\prime}\right) \leq h_{G}\left(E_{x}^{\prime}\right)$. We also note that

$$
d\left(E_{x}^{\prime}, \partial \Omega\right) \geq d\left(E_{x}^{\prime}, \partial G\right)=d\left(w_{0}, a\right) \geq d\left(E_{x}^{\prime}, \partial \Omega\right),
$$

because $a \in \partial \Omega$, and therefore $d\left(E_{x}^{\prime}, \partial \Omega\right)=d\left(E_{x}^{\prime}, \partial G\right)$ so that $J_{\Omega}\left(E_{x}^{\prime}\right)=J_{G}\left(E_{x}^{\prime}\right)$. Moreover, since the hyperbolic distance is conformally invariant, $h_{G}\left(E_{x}^{\prime}\right)=h_{G}\left(f\left(E_{x}\right)\right)=$ $h_{\mathbb{H}}\left(E_{x}\right)=h_{\mathbb{H}}\left(E^{*}\right)$. Hence, for a small enough $x>0$,

$$
\begin{aligned}
\kappa(\Omega) & \leq \frac{h_{\Omega}\left(E_{x}^{\prime}\right)}{J_{\Omega}\left(E_{x}^{\prime}\right)} \leq \frac{h_{G}\left(E_{x}^{\prime}\right)}{J_{G}\left(E_{x}^{\prime}\right)}=\frac{h_{\mathbb{H}}\left(E^{*}\right)}{\log (1+F(x))} \\
& <\frac{h_{\mathbb{H}}\left(E^{*}\right)}{\log (1+F(0))}=\frac{h_{\mathbb{H}}\left(E^{*}\right)}{J_{\mathbb{H}}\left(E^{*}\right)}=\kappa(\mathbb{H}) .
\end{aligned}
$$

The proof is finished.

Now Theorem 1.5 follows from Lemmas 3.1, 3.4 and 3.6.

\section{Extremal CONFiguration of three points in $\mathbb{H}$}

In this section, we work to find extremal configurations of three-point sets $E$ in the upper half-plane for the functional $h_{\mathbb{H}}(E) / J_{\mathbb{H}}(E)$. Since the both quantities $h_{\mathbb{H}}(E)$ and $J_{\mathbb{H}}(E)$ are invariant under the affine mappings of the form $z \mapsto a z+b$ with $a>0$ and $b \in \mathbb{R}$, we may restrict our attention to the family $\mathcal{E}$ of three-point subsets $E$ of $\mathbb{H}$ containing $i=\sqrt{-1}$ with $d(E, \partial \mathbb{H})=d(i, \partial \mathbb{H})=1$. Namely, the infimum in the definition of $\kappa_{3}(\mathbb{H})$ may be limited to $\mathcal{E}$ :

$$
\kappa_{3}(\mathbb{H})=\inf _{E \in \mathcal{E}} \frac{h_{\mathbb{H}}(E)}{J_{\mathbb{H}}(E)}=\inf _{E \in \mathcal{E}} \frac{h_{\mathbb{H}}(E)}{\log (1+d(E))} .
$$

Our goal in this section is to determine the extremal sets $E$ for which the above infimum is attained, and to compute (at least numerically) the value of $\kappa_{3}(\mathbb{H})$. First, we note the following fact for the upper half-plane $\mathbb{H}$. Though the result is essentially known (e.g., [18, Lemma $4.9(2)]$ ), we give a short proof for convenience of the reader.

\section{Lemma 4.1.}

$$
\kappa_{2}(\mathbb{H})=\inf _{z_{1}, z_{2} \in \mathbb{H}} \frac{h_{\mathbb{H}}\left(z_{1}, z_{2}\right)}{j_{\mathbb{H}}\left(z_{1}, z_{2}\right)}=1
$$

Proof. Note that $\rho_{\mathbb{H}}(z)=1 / \operatorname{Re} z=1 / d(z, \partial \mathbb{H})$. Hence, we have $h_{\mathbb{H}}(z, w)=k_{\mathbb{H}}(z, w)$ for $z, w \in \mathbb{H}$. Thus, the inequality $j_{\mathbb{H}}(z, w) \leq h_{\mathbb{H}}(z, w)$ is nothing but the Gehring-Palka inequality [5]. Hence, we have $\kappa_{2}(\mathbb{H}) \geq 1$. On the other hand, by Lemma 2.3, we have $\kappa_{2}(\mathbb{H}) \leq c(\mathbb{H}) \leq 1$, where the last inequality follows from Theorem A.

We will write

$$
\Delta\left(z_{0}, r\right)=\left\{z \in \mathbb{H}: h_{\mathbb{H}}\left(z, z_{0}\right)<r\right\}=\left\{z:\left|z-z_{0}\right|<\rho\left|z-\bar{z}_{0}\right|\right\}
$$


for the open hyperbolic disk in $\mathbb{H}$ centered at $z_{0} \in \mathbb{H}$ with hyperbolic radius $r>0$, where $\rho=\tanh (r / 2)=\left(e^{r}-1\right) /\left(e^{r}+1\right) \in(0,1)$ and denote its closure by $\bar{\Delta}\left(z_{0}, r\right)$. We need the following elementary fact for the proof of Lemma 4.15, which will be a key result below.

Lemma 4.2. Let $C$ be the boundary circle of the hyperbolic disk $\Delta\left(z_{0}, r\right)$ in $\mathbb{H}$.

(i) The Euclidean distance $\left|z-z_{0}\right|$ between $z \in C$ and $z_{0}$ takes its maximum at the top of $C$ and its minimum at the bottom of $C$.

(ii) The Euclidean diameter of the circle $C$ is $2\left(\operatorname{Im} z_{0}\right) \sinh r$.

(iii) The hyperbolic distance of the endpoints of an arbitrary diameter of the circle $C$ is at least equal to $\varphi(r)$ given in (4.3).

Proof. We write $z_{0}=x_{0}+i y_{0}$. It is well known (see, e.g., [18, (4.11)]) that the boundary of $\Delta\left(z_{0}, r\right)$ is the Euclidean circle $|z-c|=R$, where

$$
c=x_{0}+i y_{0} \cosh r \quad \text { and } \quad R=y_{0} \sinh r .
$$

Since $\operatorname{Re} z_{0}=\operatorname{Re} c$ and $\operatorname{Im} z_{0}<\operatorname{Im} c$, it is evident that $\left|z-z_{0}\right|$ is maximized at $z=c+i R$ and minimized at $z=c-i R$ on $C$. The proof of the first assertion is now complete. The second assertion is clear because the Euclidean diameter of $C$ is $2 R$. It is clear that the diameter of the circle $C$ with the minimal hyperbolic diameter is $[c-R, c+R]$. We now compute the hyperbolic distance

$$
\begin{aligned}
h_{\mathbb{H}}(c+R, c-R) & =h_{\mathbb{H}}(i \cosh r+\sinh r, i \cosh r-\sinh r) \\
& =2 \operatorname{artanh} \frac{\sinh r}{\sqrt{\cosh 2 r}} \\
& =\log \frac{\sqrt{\cosh 2 r}+\sinh r}{\sqrt{\cosh 2 r}-\sinh r} \\
& =2 \log \frac{\sqrt{\cosh 2 r}+\sinh r}{\cosh r}=: \varphi(r) .
\end{aligned}
$$

Then the third assertion follows.

Remark 4.4. By geometry, we see that $\left|c+i R e^{ \pm i \theta}-z_{0}\right|$ is strictly decreasing in $0<\theta<\pi$, which will be needed in the proof of Lemma 4.15.

We remark also that the sharp upper bound of the hyperbolic distance of the endpoints of a diameter of $C$ is $h_{\mathbb{H}}(c+i R, c-i R)=2 r$. By the form of $\varphi(r)$, we also see that $\varphi(r) \rightarrow \log \frac{\sqrt{2}+1}{\sqrt{2}-1}=2 \log (\sqrt{2}+1)=1.7627 \ldots$ as $r \rightarrow+\infty$.

In order to find the extremal configuration, we divide the family $\mathcal{E}$ into one-parameter subfamilies. More concretely, for $u>0$, let $\mathcal{E}(u)$ be the subfamily of $\mathcal{E}$ consisting of sets $E$ with $h_{\mathbb{H}}(E)=2 u$. Then

$$
\kappa_{3}(\mathbb{H})=\inf _{0<u<+\infty} \inf _{E \in \mathcal{E}(u)} \frac{2 u}{J_{\mathbb{H}}(E)}=\inf _{0<u<+\infty} \frac{2 u}{\log (1+M(u))},
$$


where

$$
M(u)=\sup _{E \in \mathcal{E}(u)} d(E)
$$

Our task is to find the extremal configuration of $E \in \mathcal{E}(u)$ for the functional $d(E)$. We first define a candidate of the extremal set. For a given number $u>0$, we choose $t>0$ and $\theta \in(0, \pi / 2)$ such that

$$
h_{\mathbb{H}}\left(i e^{t+i \theta}, i e^{t-i \theta}\right)=h_{\mathbb{H}}\left(i e^{t+i \theta}, i\right)=2 u .
$$

In other words, we choose $t$ and $\theta$ so that the set $E^{*}(u)=\left\{i, i e^{t+i \theta}, i e^{t-i \theta}\right\}$ forms the vertices of a hyperbolic equilateral triangle with sidelength $2 u$. We now give formulae describing $\theta$ and $t$ in terms of $u$. Since $h_{\mathbb{H}}\left(i e^{t+i \theta}, i e^{t}\right)=u$, we obtain $u=2 \operatorname{artanh}(\tan (\theta / 2))$ and thus

$$
\theta=2 \arctan (\tanh (u / 2)) .
$$

Moreover, by the hyperbolic cosine formula for a hyperbolic right triangle [22, Thm 7.11.1, p. 146], we have

$$
\cosh t=\cosh h_{\mathbb{H}}\left(i e^{t}, i\right)=\frac{\cosh h_{\mathbb{H}}\left(i e^{t+i \theta}, i\right)}{\cosh h_{\mathbb{H}}\left(i e^{t+i \theta}, i e^{t}\right)}=\frac{\cosh 2 u}{\cosh u} .
$$

Hence,

$$
t=\operatorname{arcosh}((\cosh 2 u) / \cosh u)
$$

We now compute

$$
\left|i e^{t+i \theta}-i e^{t-i \theta}\right|=2 e^{t} \sin \theta=\chi(u)
$$

where

$$
\begin{aligned}
\chi(u) & =2 e^{\operatorname{arcosh}((\cosh 2 u) / \cosh u)} \sin [2 \arctan \tanh (u / 2)] \\
& =2 \frac{\cosh 2 u+\sqrt{\left(\cosh ^{2} 2 u\right)-\left(\cosh ^{2} u\right)}}{\cosh u} \cdot \tanh u \\
& =\frac{2 \sinh u}{1+\sinh ^{2} u}\left[1+2 \sinh ^{2} u+\sinh u \sqrt{3+4 \sinh ^{2} u} .\right.
\end{aligned}
$$

Note that $\chi(u) \leq d\left(E^{*}(u)\right)$. In the same way, we compute

$$
\begin{aligned}
\operatorname{Im}\left(i e^{t+i \theta}\right) & =e^{t} \cos \theta=e^{\operatorname{arcosh}((\cosh 2 u) / \cosh u)} \cos [2 \arctan \tanh (u / 2)] \\
& =\frac{\cosh 2 u+\sqrt{\cosh ^{2} 2 u-\cosh ^{2} u}}{\cosh u} \cdot \frac{1}{\cosh u}>\frac{\cosh 2 u}{\cosh ^{2} u}>1 .
\end{aligned}
$$

Therefore, we obtain $d\left(E^{*}(u), \partial \mathbb{H}\right)=1$ for every $u>0$. We summarize the above observations in the following lemma.

Lemma 4.10. The set $E^{*}(u)$ of the vertices of the hyperbolic equilateral triangle in $\mathbb{H}$ with sidelength $2 u$ constructed above belongs to $\mathcal{E}(u)$ for every $u>0$. 
We make further preparatory observations.

Lemma 4.11. If $0<u \leq \log (11 / 4) \approx 1.0116$, then $d\left(E^{*}(u)\right)=\chi(u)$ and

$$
\frac{2 u}{\log (1+M(u))}<1 \text {. }
$$

Proof. We will prove the inequality

$$
\frac{2 u}{\log (1+\chi(u))}<1
$$

for $0<u \leq \log (11 / 4)$. Since $E^{*}(u) \in \mathcal{E}(u)$ by Lemma 4.10, we have $M(u) \geq d\left(E^{*}(u)\right) \geq$ $\chi(u)$. Thus, the second assertion will follow from (4.12).

By using the elementary inequality $\sqrt{3+4 \sinh ^{2} u}>\sqrt{3+3 \sinh ^{2} u}=\sqrt{3} \cosh u$ for $u>0$, we obtain the estimate

$$
\chi(u)>\frac{2 \sinh u}{1+\sinh ^{2} u}\left[1+2 \sinh ^{2} u+\sqrt{3} \sinh u \cosh u\right] .
$$

Thus, we have

$$
\begin{aligned}
\chi(u)+1-e^{2 u} & \geq \frac{2 \sinh u}{1+\sinh ^{2} u}\left[1+2 \sinh ^{2} u+\sqrt{3} \sinh u \cosh u\right]+1-e^{2 u} \\
& =\frac{(\sqrt{3}-1)\left(e^{u}+1\right)\left(e^{u}-1\right)^{2} P\left(e^{u}-1\right)}{e^{u}\left(e^{2 u}+1\right)^{2}},
\end{aligned}
$$

where $P(T)$ is the polynomial given by

$$
P(T)=4+(7+\sqrt{3}) T+4 T^{2}-\sqrt{3} T^{3}-\frac{1+\sqrt{3}}{2} T^{4} .
$$

We now estimate $P(T)$ for $T \geq 0$ from below:

$$
P(T) \geq 4+8 T+4 T^{2}-2 T^{3}-2 T^{4}=2(1+T)\left(2+2 T-T^{3}\right) .
$$

Since $Q(T)=2+2 T-T^{3}$ is concave on $[0,+\infty)$, we have

$$
Q(T) \geq \min \{Q(0), Q(7 / 4)\}=9 / 64>0 \quad \text { for } 0 \leq T \leq 7 / 4 .
$$

Hence, we have proved that $e^{2 u}<1+\chi(u)$ and thus (4.12) holds for $0<u \leq \log (11 / 4)$.

Finally, we prove that $d\left(E^{*}(u)\right)=\chi(u)$ for such $u$. Indeed, the inequality

$$
\left|i e^{t+i \theta}-i e^{t-i \theta}\right|<\left|i e^{t+i \theta}-i\right|
$$

would hold otherwise. Then the two-point subset $E=\left\{i, i e^{t+i \theta}\right\}$ of $E^{*}(u)$ satisfies $h_{\mathbb{H}}(E)=2 u, d(E)=d\left(E^{*}(u)\right)$ and $d(E, \partial \mathbb{H})=d\left(E^{*}(u), \partial \mathbb{H}\right)=1$. Thus, we would have

$$
\frac{2 u}{\log (1+\chi(u))}>\frac{2 u}{J_{\mathbb{H}}\left(E^{*}(u)\right)}=\frac{2 u}{J_{\mathbb{H}}(E)} \geq \kappa_{2}(\mathbb{H})=1
$$

by Lemma 4.1. This contradicts (4.12). In this way, we have proved that $d\left(E^{*}(u)\right)=$ $\chi(u)$. 
Lemma 4.13. Let $0<u<+\infty$. The condition $\varphi(2 u) \geq 2 u$ holds if and only if $u \leq u_{0}$, where $\varphi$ is given in (4.3) and $u_{0} \approx 0.831443$ is the positive solution to the equation $4 \cosh ^{4} u=\cosh 4 u$.

Proof. We observe that for $u>0$,

$$
\begin{aligned}
& \varphi(2 u)=2 \operatorname{artanh}[(\sinh 2 u) / \sqrt{\cosh 4 u}]<2 u \\
\Leftrightarrow & \frac{\sinh 2 u}{\sqrt{\cosh 4 u}}=\frac{2 \sinh u \cosh u}{\sqrt{\cosh 4 u}}<\tanh u=\frac{\sinh u}{\cosh u} \\
\Leftrightarrow & 4<\frac{\cosh 4 u}{\cosh ^{4} u} .
\end{aligned}
$$

Since $(\cosh 4 u) / \cosh ^{4} u$ increases from 1 to 8 when $u$ moves from 0 to $+\infty$, there exists a unique number $u_{0}>0$ satisfying the relation $4=\left(\cosh 4 u_{0}\right) / \cosh ^{4} u_{0}$. We now see that $\varphi(2 u)<2 u$ if and only if $u>u_{0}$.

The following elementary result is also needed later.

Lemma 4.14. The function $f(x)=x / \log (1+2 \sinh x)$ strictly increases from $1 / 2$ to 1 as $x$ moves from 0 to $+\infty$.

Proof. Because $f(x)=x / \log \left(e^{x}-e^{-x}+1\right)$, differentiation yields

$$
f^{\prime}(x)=h(x) /\left[\log \left(e^{x}-e^{-x}+1\right)\right]^{2}, \text { where } h(x)=\log \left(e^{x}-e^{-x}+1\right)-\frac{x\left(e^{x}+e^{-x}\right)}{e^{x}-e^{-x}+1} .
$$

Further, we have

$$
h^{\prime}(x)=-\frac{x\left(e^{x}-e^{-x}\right)}{e^{x}-e^{-x}+1}+\frac{x\left(e^{x}+e^{-x}\right)^{2}}{\left(e^{x}-e^{-x}+1\right)^{2}}=\frac{x\left(e^{-x}-e^{x}+4\right)}{\left(e^{x}-e^{-x}+1\right)^{2}}=\frac{2 x(2-\sinh x)}{(1+2 \sinh x)^{2}} .
$$

We now see that $h^{\prime}(x)>0$ for $0<x<\operatorname{arsinh} 2$ and $h^{\prime}(x)<0$ for $\operatorname{arsinh} 2<x$. Since $h(0)=0$ and

$$
h(x)=x+\log \left(1+e^{-x}-e^{-2 x}\right)-x \frac{1+e^{-2 x}}{1+e^{-x}-e^{-2 x}}=O\left(x e^{-x}\right)=o(1)
$$

as $x \rightarrow+\infty$, the function $h(x)$ is positive for all $x>0$. Hence, $f^{\prime}(x)>0$ for all $x>0$, which implies that $f(x)$ is strictly increasing in $x>0$. It is easy to see that $f(x) \rightarrow 1 / 2$ as $x \rightarrow 0$ and that $f(x) \rightarrow 1$ as $x \rightarrow+\infty$.

We are ready to prove our result.

Lemma 4.15. Let $u>0$. Then the quantity $M(u)$ defined in (4.6) is evaluated as

$$
M(u)= \begin{cases}\chi(u) & \text { if } 0<u<u_{0}, \\ 2 \sinh 2 u & \text { if } u_{0} \leq u,\end{cases}
$$


where $\chi(u)$ is given in (4.9) and $u_{0} \approx 0.831443$ is the positive solution to the equation $4 \cosh ^{4} u=\cosh 4 u$. Moreover, when $0<u<u_{0}$, a set $E \in \mathcal{E}(u)$ satisfies $d(E)=M(u)$ if and only if $E=E^{*}(u)$.

Proof. We denote the circle $\partial \Delta(i, 2 u)$ by $C$ in the following. Since every $E \in \mathcal{E}(u)$ is contained in the closed disk $\bar{\Delta}(i, 2 u)$, the diameter $d(E)$ is at most $2 \sinh 2 u$ by Lemma 4.2 (ii). Hence, we observe that

$$
M(u) \leq 2 \sinh 2 u, \quad u>0 .
$$

First, we assume that $u \geq u_{0}$; equivalently by Lemma $4.13, \varphi(2 u) \leq 2 u$. Let $z_{1}, z_{2}$ be the endpoints of the horizontal diameter of the boundary circle $C=\partial \Delta(i, 2 u)$. Note that $\operatorname{Im} z_{j}=\cosh 2 u>1$. Then, by Lemma $4.2\left(\right.$ iii), $h_{\mathbb{H}}\left(z_{1}, z_{2}\right)=\varphi(2 u) \leq 2 u$. Thus, $E=\left\{i, z_{1}, z_{2}\right\} \in \mathcal{E}(u)$ which implies $d(E)=2 \sinh 2 u \leq M(u)$. Therefore, we have proved that $M(u)=2 \sinh 2 u$. Note that the extremal set $E$ is not necessarily unique when $\varphi(2 u)<2 u$ (for instance, we can rotate the diameter a little about the Euclidean center of $C)$.

Next, we assume that $u<u_{0}$; namely, $\varphi(2 u)>2 u$. We prove that there exists a set $E_{0} \in \mathcal{E}(u)$ attaining the supremum in (4.6); namely, $M(u)=d\left(E_{0}\right)$. Indeed, by definition, we can find a sequence of sets $E_{k}$ in $\mathcal{E}(u)$ such that $d\left(E_{k}\right) \rightarrow M(u)$ as $k \rightarrow \infty$. Since each $E \in \mathcal{E}(u)$ is contained in the closed hyperbolic disk $\bar{\Delta}(i, 2 u)$, by passing to a subsequence if necessary, we may assume that $E_{k}=\left\{i, z_{k}, w_{k}\right\}$ and $z_{k} \rightarrow z_{\infty}$ and $w_{k} \rightarrow w_{\infty}$ as $k \rightarrow \infty$ for some $z_{\infty}, w_{\infty} \in \bar{\Delta}(i, 2 u)$. By continuity, we have $d\left(E_{\infty}\right)=M(u)$ for $E_{\infty}=\left\{i, z_{\infty}, w_{\infty}\right\}$. We have to check that $E_{\infty}$ belongs to $\mathcal{E}(u)$. If $E_{\infty}$ consists only of two points, by Lemma 4.1 ,

$$
\log (1+M(u)) \leq J_{\mathbb{H}}\left(E_{\infty}\right) \leq h_{\mathbb{H}}\left(E_{\infty}\right)=2 u,
$$

which contradicts Lemma 4.11 because $u \leq u_{0}<\log (11 / 4)$. We have proved the claim.

Now assume that $E_{0}=\left\{i, z_{0}, w_{0}\right\} \in \mathcal{E}(u)$ satisfies $d\left(E_{0}\right)=M(u)$. By assumption, we have $z_{0} \in \bar{\Delta}(i, 2 u) \cap \bar{\Delta}\left(w_{0}, 2 u\right)$. Observe that $z_{0} \in \partial \Delta(i, 2 u)=C$ in the present situation. In fact, let $r=h_{\mathbb{H}}\left(z_{0}, w_{0}\right)$ and suppose $h_{\mathbb{H}}\left(z_{0}, i\right)<2 u$. Then $z_{0}$ can be moved along the circle $\partial \Delta\left(w_{0}, r\right)$ upwards a bit to get a new point $z_{0}^{\prime}$ in such a way that

$$
\operatorname{Im} z_{0}<\operatorname{Im} z_{0}^{\prime}, h_{\mathbb{H}}\left(z_{0}^{\prime}, i\right)<2 u, h_{\mathbb{H}}\left(z_{0}^{\prime}, w_{0}\right)=r \quad \text { and }\left|z_{0}-w_{0}\right|<\left|z_{0}^{\prime}-w_{0}\right|
$$

by Lemma 4.2 and Remark4.4. Hence we would have $h_{\mathbb{H}}\left(E_{0}^{\prime}\right)=h_{\mathbb{H}}\left(E_{0}\right)$ and $d\left(E_{0}\right)<d\left(E_{0}^{\prime}\right)$ for $E_{0}^{\prime}=\left\{i, z_{0}^{\prime}, w_{0}\right\}$. This, however, violates the initial assumption that $d\left(E_{0}\right)=M(u)$. Therefore, we conclude that $h_{\mathbb{H}}\left(z_{0}, i\right)=2 u$. In the same way, we obtain $h_{\mathbb{H}}\left(w_{0}, i\right)=2 u$. We can further prove, as before (cf. the proof of Lemma 4.11), that $\left|z_{0}-w_{0}\right|=d\left(E_{0}\right)$.

The remaining task is now to determine the configuration of the points $z_{0}, w_{0}$ on the circle $C$ maximizing the quantity $\left|z_{0}-w_{0}\right|$ under the constraints $h_{\mathbb{H}}\left(z_{0}, w_{0}\right) \leq 2 u$ and $\min \left\{\operatorname{Im} z_{0}, \operatorname{Im} w_{0}\right\} \geq 1$. We recall that the hyperbolic distance of the endpoints of an arbitrary Euclidean diameter of $C$ is at least $\varphi(2 u)$ by Lemma 4.2(iii). We first suppose that $\varphi(2 u)<2 u$. Let $C_{0}$ be the shorter component of $C \backslash\left\{z_{0}, w_{0}\right\}$. It is evident that the 
chord $\left|z_{0}-w_{0}\right|$ is shortest when (and only when) $z_{0}$ and $w_{0}$ are situated symmetrically with respect to the imaginary axis. Therefore, we have

$$
E_{0}=E^{*}(u) \text { and } M(u)=2 u / \log \left(1+d\left(E^{*}(u)\right)\right)=\xi(u) .
$$

By the above proof, uniqueness of the extremal set for $0<u \leq u_{0}$ is clear. Thus, the proof is now complete.

Remark 4.16. In view of Lemmas 4.11 and 4.14, as a corollary of the last lemma, we have the inequality

$$
\inf _{E \in \mathcal{E}(u)} \frac{2 u}{J_{\mathbb{H}}(E)}=\frac{2 u}{\log (1+M(u))}<1
$$

for every $u>0$.

We are now in a position to prove the following theorem.

Theorem 4.17. There is a zero $u=u^{*}$ of the derivative $\xi^{\prime}(u)$ of the function

$$
\xi(u)=\frac{2 u}{\log (1+\chi(u))}
$$

in the interval $0<u<u_{0} \approx 0.83$ such that

$$
\kappa(\mathbb{H})=\frac{h_{\mathbb{H}}\left(z^{*}, w^{*}\right)}{\log \left(1+\left|z^{*}-w^{*}\right|\right)}=\frac{h_{\mathbb{H}}\left(E^{*}\right)}{\log \left(1+d\left(E^{*}\right) / d\left(E^{*}, \partial \mathbb{H}\right)\right)},
$$

where $u_{0}$ is given in Lemma 4.13, $E^{*}=E^{*}\left(u^{*}\right)=\left\{i, z^{*}, w^{*}\right\}, z^{*}=i e^{t^{*}+i \theta^{*}}, w^{*}=i e^{t^{*}-i \theta^{*}}$ and $t^{*}, \theta^{*}$ are given in (4.8) and (4.7), respectively, for $u=u^{*}$. Moreover, if $\kappa(\mathbb{H})=$ $h_{\mathbb{H}}(E) / \log (1+d(E) / d(E, \partial \mathbb{H}))$ for a three-point set $E$ in $\mathbb{H}$, then there are real numbers $a, b$ with $a>0$ such that $E=a E^{*}+b$.

Proof. Lemma 4.15 implies that for $u \geq u_{0}=0.831 \ldots$,

$$
\frac{2 u}{\log (1+M(u))}=\frac{2 u}{\log (1+2 \sinh 2 u)} .
$$

Since the function $x / \log (1+2 \sinh x)$ is increasing in $0<x<+\infty$ by Lemma 4.14, we can restrict the range of the infimum in (4.5) to $\left(0, u_{0}\right]$ :

$$
\kappa(\mathbb{H})=\kappa_{3}(\mathbb{H})=\inf _{0<u \leq u_{0}} \frac{2 u}{\log (1+M(u))}=\inf _{0<u \leq u_{0}} \frac{2 u}{\log (1+\chi(u))}=\inf _{0<u \leq u_{0}} \xi(u),
$$

where $\chi(u)$ is given in (4.9). By the form of $\chi(u)$ in (4.9), we observe that $\chi(u)=2 u+$ $2 \sqrt{3} u^{2}+O\left(u^{3}\right)$ as $u \rightarrow 0^{+}$. Thus, we obtain $\xi(u) \geq 2 u / \log (1+\chi(u))=1-(\sqrt{3}-1) u+O\left(u^{2}\right)$ as $u \rightarrow 0^{+}$. In particular, $\xi\left(0^{+}\right)=1$ and $\xi^{\prime}\left(0^{+}\right)=1-\sqrt{3}<0$. Since $\xi^{\prime}\left(u_{0}\right)=0.1917 \cdots>0$, the above infimum of $\xi(u)$ is attained at its critical point in $\left(0, u_{0}\right)$.

The last assertion easily follows from the uniqueness of the extremal set in Lemma 4.15 . The proof is now complete. 
See Figure 2 for the graph of the function $2 u / \log (1+M(u))$. By numerical computations, we obtain $u^{*} \approx 0.432335123777, t^{*} \approx 0.727535978839, \theta^{*} \approx 0.419463976058$, and $\kappa(\mathbb{H})=\xi\left(u^{*}\right) \approx 0.8750987500145$. Note that by Theorem $1.5 \kappa(\mathbb{H})=\kappa(\Omega)$ for a convex hyperbolic domain $\Omega$. In conclusion, we have the following theorem:

Theorem 4.18. For any convex hyperbolic domain $\Omega, \kappa(\Omega) \approx 0.875098750014$.

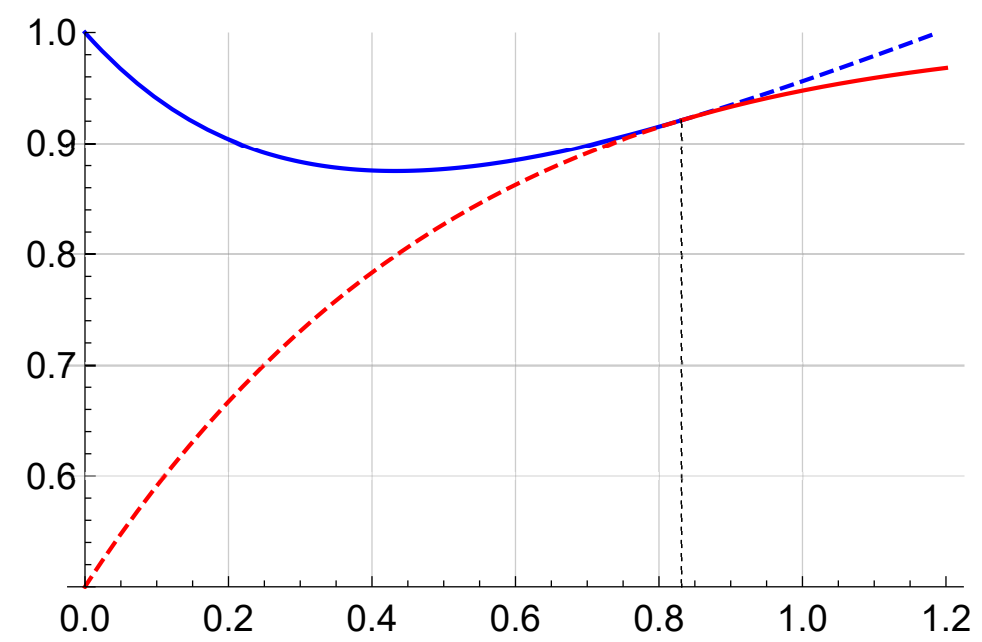

FiguRE 2. The graph of $2 u / \log (1+M(u))$ (the thick line); the blue curve indicates the graph of $\xi(u)$ and the red one does the graph of $2 u / \log (1+$ $2 \sinh 2 u$ )

Finally, we prove Theorem 1.6.

Proof of Theorem 1.6. It remains to prove the first assertion. Let $\Omega \subsetneq \mathbb{C}$ be a convex domain and suppose that $\kappa(\Omega)=h_{\Omega}(E) / J_{\Omega}(E)$ for a compact subset $E$ of $\Omega$. As in the proof of Lemma 2.1. we take points $z_{0}, z_{1}, z_{2} \in E$ so that $d(E)=\left|z_{1}-z_{2}\right|$ and $d(E, \partial \Omega)=d\left(z_{0}, \partial \Omega\right)$ and let $E_{0}=\left\{z_{0}, z_{1}, z_{2}\right\}$. (Since $\kappa(\Omega)=\kappa(\mathbb{H})<1$, the set $E_{0}$ contains exactly three points.) By Lemma 2.1 , we have $\kappa_{3}(\Omega)=\kappa(\Omega)$. Thus, in the chain of inequalities (2.2), the last term is the same as the initial term. Thus, we have $h_{\Omega}(E)=h_{\Omega}\left(E_{0}\right)$. Hence $\kappa(\Omega)=h_{\Omega}\left(E_{0}\right) / J_{\Omega}\left(E_{0}\right)$.

Let $\zeta_{0} \in \partial \Omega$ be such that $d\left(E_{0}, \partial \Omega\right)=d\left(z_{0}, \partial \Omega\right)=\left|z_{0}-\zeta_{0}\right|$. Take a half-plane $H$ as in the proof of Lemma 3.4 such that $\Omega \subset H$ and $z_{0} \in \partial H$. Then $J_{\Omega}\left(E_{0}\right)=J_{H}\left(E_{0}\right)$ and $h_{\Omega}\left(E_{0}\right) \geq h_{H}\left(E_{0}\right)$. If $\Omega$ is a proper subdomain of $H$, then we would have $h_{H}\left(E_{0}\right)<h_{\Omega}\left(E_{0}\right)$. Thus,

$$
\kappa(H) \leq \frac{h_{H}\left(E_{0}\right)}{J_{H}\left(E_{0}\right)}<\frac{h_{\Omega}\left(E_{0}\right)}{J_{\Omega}\left(E_{0}\right)}=\kappa(\Omega) .
$$

On the other hand, Theorem 1.5 yields $\kappa(H)=\kappa(\Omega)$, which is a contradiction. Thus, $\Omega$ equals $H$, a half-plane. 


\section{Application to Capacity Estimation}

Finally, we apply the results above to capacity estimation. First, we recall some basic notions.

Definition 5.1. [18, Def. 9.2, p. 150] A pair $(\Omega, E)$ of a domain $\Omega$ in $\mathbb{C}$ and a non-empty compact subset $E$ of $\Omega$ is called a condenser. The capacity of this condenser is defined to be

$$
\operatorname{cap}(\Omega, E)=\inf _{u} \iint_{\mathbb{C}}|\nabla u(z)|^{2} d x d y \quad(z=x+i y),
$$

where the infimum is taken over the family of all non-negative functions $u$ in the Sobolev class $W_{\text {loc }}^{1,2}(\mathbb{C})$ with compact support in $\Omega$ such that $u(z) \geq 1$ for $z \in E$.

If $\Omega$ is a simply connected proper subdomain of $\mathbb{C}$ and $E$ is a (non-degenerate) continuum in $\Omega$ such that the set $R=\Omega \backslash E$ is a doubly connected domain (a ring), then its modulus is known to be $2 \pi / \operatorname{cap}(\Omega, E)$.

We define the homeomorphism $\mu:(0,1) \rightarrow \mathbb{R}^{+}$by the formula (see, e.g., [18, 7.4.1, p. 122])

$$
\mu(r)=\frac{\pi}{2} \cdot \frac{\mathcal{K}\left(\sqrt{1-r^{2}}\right)}{\mathcal{K}(r)},
$$

where $\mathcal{K}(r)$ is Legendre's complete elliptic integral of the first kind defined by

$$
\mathcal{K}(r)=\int_{0}^{1} \frac{d x}{\sqrt{\left(1-x^{2}\right)\left(1-r^{2} x^{2}\right)}} .
$$

It is known that $\mu(r)$ represents the modulus of the Grötzsch ring $\mathbb{D} \backslash[0, r]$. In particular, $\mu(r)$ decreases from $+\infty$ to 0 as $r$ moves from 0 to 1 . We note that $2 \pi / \mu(r)$ is the capacity of $\mathbb{D} \backslash[0, r]$. For later convenience, we put

$$
\Phi(x)=\frac{2 \pi}{\mu(\tanh (x / 2))}, \quad 0<x<\infty .
$$

Note that $\Phi(x)$ increases from 0 to $+\infty$ as $x$ moves from 0 to $+\infty$. We are ready to give the main result in this section. Recall that $J_{\Omega}(E)=\log (1+d(E) / d(E, \partial \Omega))$.

Theorem 5.2. Let $E$ be a continuum in a simply connected domain $\Omega \subsetneq \mathbb{C}$. Then the following are valid.

(i) The inequality

$$
\operatorname{cap}(\Omega, E) \geq \Phi\left(\kappa(\Omega) J_{\Omega}(E)\right) \geq \Phi\left(\kappa_{0} J_{\Omega}(E)\right)
$$

holds, where $\kappa_{0}$ is given in (1.7).

(ii) If $\Omega$ is convex,

$$
\left.\operatorname{cap}(\Omega, E) \geq \Phi\left(\kappa_{1} J_{\Omega}(E)\right)\right),
$$

where $\kappa_{1}=\kappa(\mathbb{D})>0.87509875$. 
Proof. Let $f: \Omega \rightarrow \mathbb{D}$ be a conformal homeomorphism and set $E^{\prime}=f(E)$. Since the capacity and the hyperbolic distance are conformally invariant, we obtain

$$
\operatorname{cap}(\Omega, E)=\operatorname{cap}\left(\mathbb{D}, E^{\prime}\right) \geq \Phi\left(h_{\mathbb{D}}\left(E^{\prime}\right)\right)=\Phi\left(h_{\Omega}(E)\right),
$$

where we used a consequence of the circular symmetrization (see [18, Lemma 9.20, p. 163]). Other parts follow from Corollary 1.8 and Theorem 4.18.

Example 5.3. Consider next an example where $\Omega=\{z:-1<\operatorname{Im} z<1\}$ and $E=[1,2]$. Because $\Omega$ is convex, it follows from Theorem 5.2 that

$$
\operatorname{cap}(\Omega, E) \geq \Phi\left(\kappa_{1} J_{\Omega}(E)\right) \approx \frac{2 \pi}{\mu(0.43754937 \log 2))}>2.4288 .
$$

By applying the circular (spherical) symmetrization (see [18, 9.1, pp. 155-157]) with the origin as a center and $x$-axis as the symmetrization axis. Observe first that the negative $x$-axis is contained in the complement of the symmetrized condenser whereas $[1,2]$ remains invariant and hence

$$
\operatorname{cap}(\Omega, E) \geq \tau_{2}(1)=2,
$$

where $\tau_{2}(t)$ denotes the capacity of the Teichmüller ring $\mathbb{C} \backslash([-1,0] \cup[t,+\infty))$ for $t>0$ (see [18, 7.3, pp. 120]), which is a weaker lower bound for the capacity than what we proved above. On the other hand, if we take into account that the whole left half-plane is contained in the complement of the symmetrized condenser, we obtain

$$
\operatorname{cap}(\Omega, E) \geq \Phi(\log 2)=\frac{2 \pi}{\mu(\tanh (\log \sqrt{2}))} \approx 2.55852 .
$$

Hence the value of our bound given in Theorem 5.2 lies between these two bounds obtained by symmetrization. Finally, let us find the exact value of cap $(\Omega, E)$. Obviously, $\operatorname{cap}(\Omega, E)=\operatorname{cap}\left(\Omega, E_{0}\right)$, where $E_{0}=[0,1]$. Note that the function $f(z)=\frac{2}{\pi} \log \frac{1+z}{1-z}$ maps the unit disk $\mathbb{D}$ onto $\Omega$ and that $f^{-1}\left(E_{0}\right)=[0, \tanh (\pi / 4)]$. Thus

$$
\operatorname{cap}(\Omega, E)=\operatorname{cap}\left(\Omega, E_{0}\right)=\operatorname{cap}(\mathbb{D},[0, \tanh (\pi / 4)])=\frac{2 \pi}{\mu(\tanh (\pi / 4))}=\Phi\left(\frac{\pi}{2}\right) \approx 3.75108 .
$$

Acknowledgements. The authors would like to thank the referee for detailed and constructive corrections.

\section{REFERENCES}

1. A. F. Beardon And D. Minda, The hyperbolic metric and geometric function theory, Proc. International Workshop on Quasiconformal Mappings and their Applications (IWQCMA05), eds. S. Ponnusamy, T. Sugawa and M. Vuorinen (2006), 9-56.

2. L. KEEN AND N. LAKIC, Hyperbolic geometry from a local viewpoint. London Mathematical Society Student Texts, 68. Cambridge University Press, Cambridge, 2007.

3. A. F. Beardon and Ch. Pommerenke, The Poincaré metric of plane domains, J. London Math. Soc. (2) 18 (1978), 475-483. 
4. F.W. Gehring And K. Hag, The ubiquitous quasidisk. With contributions by Ole Jacob Broch. Mathematical Surveys and Monographs, 184. American Mathematical Society, Providence, RI, 2012. xii+171 pp.

5. F.W. Gehring And B. P. Palka, Quasiconformally homogeneous domains, J. Analyse Math. 30 (1976), 172-199.

6. T. Sugawa And M. Vuorinen, Some inequalities for the Poincaré metric of plane domains. Math. Z. 250 (2005), no. 4, 885-906.

7. R. Harmelin and D. Minda, Quasi-invariant domain constants, Israel J. Math. 77 (1992), 115127.

8. D. Mejía AND D. Minda, Hyperbolic geometry in k-convex regions, Pacific J. Math. 141 (1990), $333-354$.

9. F. G. Avkhadiev And K.-J. Wirths, Schwarz-Pick type inequalities. Frontiers in Mathematics. Birkhäuser Verlag, Basel, 2009. viii+156 pp.

10. J.B. Garnett and D.E. Marshall, Harmonic measure. Cambridge Univ. Press 2005, xvi+571pp.

11. Ch. Pommerenke, Uniformly perfect sets and the Poincaré metric, Arch. Math. 32 (1979), 192-199.

12. Ch. Pommerenke, On uniformly perfect sets and Fuchsian groups, Analysis 4 (1984), 299-321.

13. T. SugaWA, Various domain constants related to uniform perfectness, Complex Variables Theory Appl. 36 (1998), 311-345.

14. T. SugaWA, Uniformly perfect sets: analytic and geometric aspects (Japanese), Sugaku 53 (2001), 387-402, English translation in Sugaku Expo. 16 (2003), 225-242.

15. M. BRIDGEMAN AND R. D. CANARY, Uniformly perfect domains and convex hulls: improved bounds in a generalization of a theorem of Sullivan. Pure Appl. Math. Q. 9 (2013), no. 1, 49-71.

16. R. Stankewitz, T. Sugawa And H. Sumi, Hereditarily non uniformly perfect sets, Disc. Cont. Dyn. Sys. 12 (8) (2019), 2391-2402.

17. X. WANG AND Q. ZHOU, Quasimöbius maps, weakly quasimöbius maps and uniform perfectness in quasi-metric spaces, Ann. Acad. Sci. Fenn. Math. 42 (2017), 257-284.

18. P. Hariri, R. Klén, and M. VuORInen, Conformally Invariant Metrics and Quasiconformal Mappings, Springer Monographs in Mathematics, Springer, Berlin, 2020.

19. F.W. Gehring And B. G. OsGood, Uniform domains and the quasi-hyperbolic metric, J. Analyse Math. 36 (1979), 50-74.

20. A. Golberg, T. Sugawa, And M. Vuorinen, Teichmüller's theorem in higher dimensions and its applications. Comput. Methods Funct. Theory, 20 (2020), 539-558.

21. F. R. KeOGh, A characterization of convex domains in the plane, Bull. London Math. Soc. 8 (1976), $183-185$.

22. A. F. Beardon, The geometry of discrete groups. Graduate texts in Math., Vol. 91, Springer-Verlag, New York, 1983.

Department of Mathematics and Statistics, University of Turku, Fi-20014 Turku, FinLAND

Email address: ormrai@utu.fi

Graduate School of Information Sciences, Tohoku University, Aoba-ku, Sendai 9808579, JAPAN

Email address: sugawa@math.is.tohoku.ac.jp

Department of Mathematics and Statistics, University of Turku, FI-20014 Turku, FinLAND

Email address: vuorinen@utu.fi 